\title{
L'aliénation parentale: points de vue et besoins d'intervenants et intervenantes
}

\author{
Marie-Hélène Gagné, Valérie Duquet et Roseline Jean \\ École de psychologie, Université Laval \\ Julie Nadeau \\ École de service social, Université Laval
}

\begin{abstract}
RÉSUMÉ
Cette étude qualitative explore les points de vue d'intervenantes et intervenants psychosociaux concernant l'aliénation parentale (AP), une problématique qui renvoie aux situations où un enfant rejette l'un de ses parents sous l'influence de son autre parent. Les participants et participantes sont des intervenants et intervenantes provenant d'organismes communautaires et d'établissements du réseau de la santé et des services sociaux œuvrant auprès de femmes, d'hommes, d'enfants et de familles. Quatre groupes focalisés ont été animés selon une méthode semi-structurée pour une durée approximative de 2 heures. Les résultats indiquent que tous les intervenants et intervenantes reconnaissent l'existence du phénomène de l'AP. De plus, il y a plus de convergences qu'attendu dans les discours des différents groupes rencontrés. Aussi, chaque groupe d'intervenants et intervenantes contribue à la compréhension d'une facette importante de l'AP. Ces résultats commandent l'émergence de recherches futures plus spécifiques qui pourraient permettre de mieux comprendre et connaître l'AP. Il serait alors possible d'aider les familles concernées par ce problème à l'aide d'interventions davantage adaptées à leurs besoins.
\end{abstract}

Depuis quelques décennies et à l'instar des autres pays développés, la proportion de familles biparentales intactes au Québec est en baisse. Elle est passée de 80,9\% en 1987 à 66,1\% en 2004 (Institut de la statistique du Québec, 2001, 2004). On sait que la séparation des conjoint(e)s peut

Cette étude a bénéficié du soutien du FQRSC via les subventions d'infrastructure octroyées au centre de recherche JEFAR et au Centre jeunesse de Québec-Institut universitaire (CJQ-IU). Elle a été réalisée avec la collaboration de plusieurs organisations de la région de Québec (CJQ-IU, Centres de santé et de services sociaux, organismes communautaires). Les auteures remercient Sylvie Drapeau, Marie-Christine Saint-Jacques et Geneviève Lessard pour leur contribution à l'interprétation des résultats, de même que Laurence Fortin-Pellerin pour son soutien technique dans l'analyse qualitative. Toute correspondance concernant cet article devra être envoyée à Marie-Hélène Gagné, professeure agrégée, École de psychologie, pavillon F.-A.-Savard, 2325, rue des Bibliothèques, Université Laval, Québec (Québec), G1V 0A6; mariehelene.gagne@psy.ulaval.ca 
contribuer à accentuer les conflits déjà existants au sein du couple ou en susciter de nouveaux. De tels conflits ont des répercussions importantes pour les enfants, surtout s'ils perdurent (Drapeau, Gagné, \& Hénault, 2004). C'est dans un tel contexte que la probabilité de voir émerger l'aliénation parentale (AP) serait accrue.

L'AP renvoie aux situations où un enfant rejette l'un de ses parents en le dénigrant de façon injustifiée sous l'influence de son autre parent (Gagné, Drapeau, \& Hénault, 2005). Ce phénomène surviendrait typiquement dans un contexte de disputes pour la garde de l'enfant (Gardner, 2003). Bien que les modèles théoriques et les études empiriques sur ce sujet soient presque inexistants et que les points de vue concernant l'AP soient diversifiés et parfois même contradictoires, ce concept influence déjà les pratiques légales et sociales. Pourtant, les discours et les besoins des différents intervenantes et intervenants concernés n'ont pas été systématiquement documentés. C'est le but poursuivi par la présente étude, qui vise à mettre en lumière les points de vue de différents groupes d'intervenants et intervenantes quant à leur manière de voir l'AP, leur conception des enjeux que l'AP soulève et leurs besoins en lien avec cette problématique.

\section{RECENSION DES ÉCRITS}

Les écrits empiriques, cliniques et théoriques au sujet de l'AP mettent en lumière les nombreuses controverses qui entourent ce construit. Ces controverses touchent à la définition et la nature de l'AP, à l'ampleur du problème et au genre du principal parent aliénant, aux fausses allégations y étant associées ainsi qu'aux modes d'intervention à privilégier.

\section{La controverse conceptuelle}

L'une des controverses entourant la problématique de l'AP concerne sa définition: s'agit-il d'un syndrome qui affecte l'enfant ou d'une problématique familiale de nature systémique? C'est à Gardner (1992, 2002) que l'on doit l'expression syndrome d'aliénation parentale (SAP), dont les manifestations les plus évidentes seraient une campagne de dénigrement injustifiée de l'enfant envers le parent aliéné qui mènerait au rejet de ce dernier. Cette campagne résulterait d'une programmation de l'enfant induite par le parent aliénant. Elle aurait pour but d'éloigner l'enfant de son autre parent.

Un élément de la controverse conceptuelle renvoie au fait de considérer l'AP comme un syndrome, ou non. Les défenseurs de l'option syndrome perçoivent l'AP comme un trouble de l'enfance dont le diagnostic est fait à partir des symptômes ${ }^{1}$ présentés par l'enfant (Gardner, 2002). Le diagnostic s'applique à l'enfant, même si la contribution d'un parent dit «aliénant » est reconnue. Kelly et Johnston (2001) préfèrent éviter le terme syndrome et parler d'enfant aliéné. Elles croient que l'enfant peut développer une animosité injustifiée, des peurs et des croyances négatives envers un parent même en l'absence d'un comportement aliénant de la part de l'autre parent (Johnston, 1993). Elles considèrent qu'il existe plusieurs facteurs prédisposants à l'AP comme des conflits conjugaux intenses et répétés avant et après la séparation ou une séparation humiliante, lorsqu'un(e) des deux conjoint(e)s est laissé pour quelqu'un(e) d'autre, par exemple. Certains facteurs pourraient influencer la réponse de l'enfant 


\section{L'ALIÉNATION PARENTALE}

à de telles situations comme les croyances et les comportements négatifs du parent aliénant, la réaction du parent rejeté ou la vulnérabilité, l'âge et le tempérament de l'enfant. La réponse affective et comportementale de l'enfant aurait à son tour une répercussion sur ses parents et sur le fonctionnement familial, ce qui créerait une dynamique circulaire.

Dans l'état très embryonnaire des connaissances actuelles, le terme $A P$ nous paraît plus approprié parce qu'il est plus englobant que le $S A P$. Il permet de concevoir ce phénomène dans un système familial caractérisé par des enjeux de pouvoir entre ses membres et de le situer dans un contexte social et culturel. De fait, des enjeux politiques, socioculturels et institutionnels peuvent accentuer le risque d'AP, qui est alors vue comme une réaction pathologique de la famille à un contexte social non favorable (Gagné et al., 2005). Cette perspective écologique donne une vision globale des facteurs qui interagissent dans l'étiologie du problème.

\section{La controverse de l'ampleur et du genre}

Malgré que l'AP soit généralement définie comme un trouble de l'enfance ou une problématique familiale, sa prévalence a plutôt été évaluée sous l'angle des conduites parentales aliénantes, des tactiques ayant pour but ou pour effet de détourner l'enfant de l'un de ses parents et de miner leurs relations. Ce glissement conceptuel a pu amener certains experts et expertes à surestimer la prévalence de l'AP et à surévaluer la responsabilité des mères dans l'apparition de cette problématique. Par exemple, sur la base de son expérience clinique, Gardner $(1992,1998)$ a soutenu que $85 \%$ à $90 \%$ des disputes concernant la garde des enfants après la séparation impliquent le syndrome d'AP et que, dans $80 \%$ à $90 \%$ des cas, la mère est le parent aliénant. Un autre exemple est l'étude descriptive de Clawar et Rivlin (1991). Sur une période de 12 ans, ces chercheurs ont étudié 700 familles séparées dont les enfants étaient âgés entre 0 et 20 ans, référées pour médiation, évaluation ou thérapie familiale. Ils concluent que $80 \%$ de ces familles présentent un profil d'AP, la moitié des cas impliquant des conduites parentales aliénantes quotidiennes. Les diverses tactiques aliénantes identifiées dans l'étude (brainwashing techniques) étaient utilisées par $4 \%$ à $85 \%$ des mères contre $2 \%$ à $25 \%$ des pères.

Les autres études sur l'AP publiées à ce jour (Baker, 2006; Dunne \& Hedrick, 1994; Lampel, 1996; Siegel \& Langford, 1998; Vassiliou \& Cartwright, 2001) ne permettent ni d'estimer la prévalence du problème ni de statuer sur le genre du principal parent aliénant. Elles ont été menées auprès de très petits échantillons et sont limitées par des problèmes de fidélité et de validité dans l'identification des cas d'AP. Cependant, un examen de la littérature sur les conflits post-séparation et sur l'adaptation des enfants à la séparation de leurs parents suggère que la prévalence de l'AP serait beaucoup moins élevée que les chiffres précédemment avancés (Campbell, 2005; Emery, 1999; Johnston \& Roseby, 1997). Non seulement les jeunes de parents séparés formeraient rarement des alliances prononcées avec un parent, mais leur préférence irait aussi souvent à leur père qu'à leur mère (Gagné, Drapeau, Melançon, Saint-Jacques, \& Lépine, 2007). Dans ses derniers écrits, Gardner (2002) observe que les pères sont désormais aussi nombreux que les mères à se montrer aliénants. Il explique cela par les contacts accrus des pères avec leurs enfants, notamment grâce aux gardes partagées. 


\section{Les fausses allégations d'abus sexuel}

Les écrits de Gardner (1998, 2002) suggèrent que les fausses allégations d'abus sexuels sont l'un des moyens utilisés par la mère aliénante pour discréditer le père des enfants; ainsi, de telles allégations seraient suspectes lorsqu'elles surviennent dans un contexte de litige pour la garde des enfants. Selon O'Leary et Moerk (1999), ce raisonnement peut être préjudiciable aux enfants de familles séparées qui sont réellement victimes d'abus sexuels, d'autant plus que le « diagnostic » d'AP souffre de sérieux problèmes de fidélité et de validité (Gagné et al., 2005). Renversant le raisonnement de Gardner, Faller (1998) a fait une démonstration convaincante que plusieurs symptômes du SAP décrits par Gardner sont aussi des signes reconnus de la présence d'abus sexuel. Le risque de confondre ces deux problématiques est donc très important.

Malgré toute l'attention accordée à ce phénomène, les études suggèrent que les fausses allégations de maltraitance ne sont pas très fréquentes. Avec des données recueillies dans le cadre de la Canadian Incidence Study of Reported Child Abuse and Neglect qui documente 7672 signalements retenus dans 51 services de protection canadiens, Trocmé et Bala (2005) montrent que seulement $4 \%$ des allégations de maltraitance étaient clairement fabriquées. Ce résultat appuie ceux d'autres études (Brown \& Frederico, 2000; Thoennes \& Tjaden, 1990). Quoique les fausses allégations soient surreprésentées chez les familles où il y a des disputes pour la garde des enfants (12\% vs $3 \%)$, le motif le plus fréquent n'est pas l'abus sexuel mais bien la négligence, et de telles allégations sont le plus souvent faites par des signalants anonymes ou par le parent non gardien, habituellement le père (Trocmé \& Bala).

\section{L'intervention}

Différentes interventions sont proposées dans la littérature afin d'aider les familles qui vivent de l'AP. Elles vont d'une position punitive (Gardner, 1998) à une position systémique (Johnston, Walters, \& Friedlander, 2001) en passant par la médiation (Vestal, 1999) et le counseling (Ellis, 2005). Certains experts et expertes se sont prononcés sur la question à partir de leur expérience professionnelle et soutiennent que les interventions de nature psychosociale doivent se faire en lien étroit avec le tribunal, puisqu'il a le pouvoir d'obliger les parents à respecter une ordonnance (Gardner, 1999; Kelly \& Johnston, 2001; Sullivan \& Kelly, 2001; Van Gijseghem, 2005). Cependant, les experts et expertes ne s'entendent pas à savoir quelle serait l'ordonnance appropriée concernant la garde de l'enfant en situation d'AP. Gardner (1998) considère que laisser l'enfant en compagnie du parent aliénant lui est préjudiciable, et il recommande que sa garde revienne au parent aliéné, même s'il faut obliger l'enfant. D'autres auteurs, comme Stahl (1999), croient qu'une telle « parentectomie » peut être préjudiciable à l'enfant et recommandent plutôt une garde partagée. Étant donné qu'aucune intervention n'a été systématiquement évaluée jusqu'à maintenant, il est impossible d'identifier l'approche qui obtient les meilleurs résultats.

\section{But et objectifs de la présente étude}

Malgré toutes ces controverses, l'AP est déjà entrée dans les pratiques légales et sociales et dans le discours de divers groupes d'intérêt. Il importe de ne pas nier le problème d'emblée, mais de l'étudier davantage. Le développement d'un discours plus scientifique relativement à l'AP pourrait réduire les 


\section{L'ALIÉNATION PARENTALE}

controverses existantes et atténuer les débats idéologiques que suscite cette problématique. La présente étude, qualitative et exploratoire, vise à documenter une variété de points de vue sur l'AP chez diverses catégories d'intervenants et intervenantes concernés par le phénomène. Plus spécifiquement, les objectifs suivants sont poursuivis: (a) explorer les conceptions de l'AP chez divers intervenants et intervenantes psychosociaux qui œuvrent auprès des femmes, des hommes, des jeunes ou des familles, dans des organismes communautaires ou des Centres de santé et de services sociaux (CSSS); (b) identifier les principaux enjeux soulevés par la reconnaissance professionnelle et sociale de l'AP pour les différents acteurs familiaux concernés; (c) identifier les préférences des intervenants et intervenantes en matière d'intervention dans ce genre de cas, de même que leurs besoins pour supporter leurs interventions; (d) mettre en lumière les convergences et les divergences dans les discours des intervenants et intervenantes concernant tous les points précédemment mentionnés, afin de situer les lieux d'harmonie et de conflits par rapport à l'AP.

\section{MÉTHODOLOGIE}

La méthodologie qualitative utilisée dans la présente étude correspond à ce que Merriam (2002) et ses collègues nomment basic interpretative research. Cette méthode descriptive et inductive vise à comprendre la signification donnée par certains individus à un phénomène. La méthodologie s'inspire aussi de l'analyse des besoins (Witkin, 1984), dans la mesure où l'on vise à cerner les besoins des intervenants et intervenantes par rapport à l'AP, en matière de savoir et de savoir-faire.

\section{Échantillon}

L'échantillon compte 25 intervenantes et intervenants répartis en quatre groupes, selon qu'ils travaillent auprès de femmes/mères ( $n=6$ femmes, milieu communautaire), d'hommes/pères $(n=7$ hommes et 1 femme, milieux communautaire et des services sociaux), d'enfants ( $n=4$ femmes et 1 homme, protection de la jeunesse) ou de familles dans leur ensemble ( $n=6$ femmes, milieu communautaire). Le choix de ces quatre catégories est basé sur le principe de diversification externe, qui implique de former autant de groupes que nécessaire pour représenter tous les acteurs concernés par la problématique étudiée. Cela favorise l'atteinte de la saturation empirique, qui survient lorsque les informations recueillies ne permettent plus d'apporter d'éléments nouveaux au corpus de données. L'échantillon répond aux critères de l'échantillon par contrastesaturation, qui permet les analyses comparatives intergroupes (Pires, 1997).

Dans l'ensemble, les intervenantes et intervenants de ces quatre groupes sont âgés entre 20 ans et 62 ans. Leurs années d'expérience en intervention varient entre 1 an et 28 ans; 21 intervenants et intervenantes détiennent un diplôme universitaire, 3 détiennent un diplôme collégial et 1 possède un diplôme professionnel. La surreprésentation des femmes reflète la réalité de l'intervention psychosociale et communautaire; ainsi, elle ne doit pas nécessairement être vue comme une limite de l'étude.

\section{Stratégie de recrutement}

Des organismes et établissements des régions de Québec et Chaudière-Appalaches ont été ciblés à partir du membership de regroupements jugés pertinents en regard de l'objet d'étude et des listes de 
ressources existantes. Les responsables des organismes (directrice ou directeur, coordonnatrice ou coordonnateur) ont d'abord été sollicités par téléphone par la responsable du projet, puis plus officiellement par écrit. Certains responsables contactés ont proposé d'autres organismes à solliciter, ce qui a entraîné un effet « boule de neige » pour le recrutement.

\section{Collecte de données et matériel}

Pour recueillir les données, la méthode du groupe focalisé a été privilégiée puisque c'est un bon moyen de comparer l'ensemble des réalités personnelles (Krueger \& Casey, 2000) et d'analyser des besoins (Simard, 1989). Cette méthode comporte certaines caractéristiques qui influencent la nature des données, dont la dynamique de groupe. Les interactions possibles entre les participants et participantes peuvent faire en sorte que des idées demeurent incomplètes ou soient coupées au fil de la discussion, impliquant une perte de données (Ritchie \& Lewis, 2003). En contrepartie, les interactions entre les participants et participantes peuvent aussi générer des informations qui ne seraient pas ressorties en entrevue individuelle (Morgan, 1988).

Le matériel comprend un questionnaire sociodémographique qui vise à recueillir des informations sur les participants et participantes, de même qu'un guide d'entrevue de groupe semi-structuré (Greenbaum, 1998) contenant six grandes questions ouvertes qui portent sur la conception de l'AP, les enjeux de la reconnaître comme une problématique réelle et importante, les enjeux de ne pas la reconnaître comme telle, les interventions réalisées en situation d'AP, la réflexion sur d'éventuelles interventions et les besoins des intervenants et intervenantes. Diverses sous-questions étaient aussi prévues et à utiliser au besoin pour relancer la discussion ou approfondir certains aspects.

\section{Procédures}

La décision de participer à cette étude s'est prise sur une base volontaire. Les organismes participants ont reçu une compensation monétaire de $60 \$$ par intervenante ou intervenant libéré, à l'exception du Centre jeunesse de Québec qui était un partenaire financier du projet.

Les quatre groupes focalisés ont été co-animés par un membre de l'équipe de recherche et une modératrice expérimentée. Leur rôle consistait à conduire et stimuler la discussion entre les participants et participantes à l'aide des questions du guide d'entrevue. Les rencontres ont eu lieu à l'Université Laval, à l'exception du groupe en protection de la jeunesse qui s'est tenu dans les locaux du Centre jeunesse de Québec. Lors des rencontres, on a rappelé aux participants et participantes les objectifs de la recherche. Le déroulement de la rencontre leur a été expliqué afin de faciliter la cueillette d'informations. Ensuite, ils ont signé un formulaire de consentement.

Chaque entrevue a duré approximativement 2 heures et a été enregistrée. Les participants et participantes ont rempli individuellement un questionnaire sociodémographique à la fin de la rencontre. Par la suite, il y a eu retranscription verbatim des résultats des entrevues pour fins d'analyse. Chaque intervenante participante ou intervenant participant a reçu un rapport synthèse de la recherche. Toutes ces procédures ont été approuvées par le Comité d'éthique de la recherche du Centre jeunesse de Québec-Institut universitaire (projet \#2005-16, accepté de 2 février 2006). 


\section{L'ALIÉNATION PARENTALE}

\section{Analyses}

Pour faire l'analyse qualitative des données recueillies, l'approche du whole group analysis a été utilisée. Elle traite les informations produites par un groupe comme une unité, sans personnaliser la contribution de chaque participant ou participante (Ritchie \& Lewis, 2003). L'analyse de contenu, basée sur l'application d'un système de codification de données qualitatives, a été privilégiée. Cette analyse est supportée par le logiciel NVivo.

Une analyse de contenu par catégorisation (L'Écuyer, 1990) a identifié les principaux thèmes abordés par les participants et participantes en regard de l'objet d'étude. Ce type d'analyse implique la lecture préliminaire des transcriptions d'entrevues et la classification des « unités de sens » (phrases ou idées) qui se ressemblent à l'intérieur de catégories conceptuelles. Si certaines de ces catégories étaient prédéterminées sur la base du guide d'entrevue, d'autres se sont développées de manière inductive et le système de classification s'est raffiné en cours d'analyse (modèle mixte d'analyse de contenu). Finalement, les similarités et les particularités de chaque groupe focalisé ont été identifiées en juxtaposant les contenus de chaque catégorie conceptuelle pour chacun des quatre groupes.

\section{RÉSULTATS ET DISCUSSION}

Cette recherche a permis de répondre à tous les objectifs de départ, soit: (a) explorer les conceptions de l'AP chez divers intervenants et intervenantes psychosociaux; (b) identifier les principaux enjeux soulevés par la reconnaissance professionnelle et sociale de l'AP; (c) identifier les préférences des intervenants et intervenantes en matière d'intervention, de même que leurs besoins pour supporter leurs interventions; (d) mettre en lumière les convergences et les divergences dans les discours des intervenants et intervenantes. Puisque ce dernier objectif est transversal à tous les thèmes abordés, il sera intégré aux trois autres à l'intérieur de cette partie du texte. De plus, vu la nature qualitative des données, les résultats et les éléments de discussion et d'interprétation y seront amalgamés afin d'éviter le morcellement de la réflexion.

\section{Conceptions de l'AP}

L'analyse a permis de faire ressortir certains éléments convergents d'une conception de l'AP. L'AP serait un problème d'ordre systémique lié à une séparation ou un divorce conflictuel. Elle impliquerait qu'un parent dénigre l'autre parent de manière injustifiée devant l'enfant. Ce dénigrement peut être associé à d'autres stratégies aliénantes qui résulteraient en une programmation de l'enfant, qu'elle soit intentionnelle ou non. Des exemples de telles stratégies sont des questions tendancieuses posées à l'enfant afin qu'il perçoive l'autre parent comme incompétent, des menaces ou des promesses faites à l'enfant afin qu'il choisisse le parent aliénant, etc. L'enfant est alors incité à dénigrer le parent rejeté en utilisant les mots du parent aliénant:

Le fond est toujours le même: c'est de dénigrer l'autre parent puis de le mettre hors circuit.

Avant de devenir aliéné, l'enfant serait au cœur d'un conflit de loyauté, ce qui l'amènerait à prendre parti pour l'un de ses parents: 
Puis un moment donné l'enfant est comme piégé entre les deux [...] l'enfant vit le conflit entre les deux parents.

Après avoir vécu cette ambivalence, l'enfant aliéné rejetterait l'un des deux parents de façon définitive. Le fait que tous les groupes d'intervenants et intervenantes aient mentionné ces aspects permet de croire que ces éléments définitionnels sont au cœur des représentations professionnelles de l’AP. On retrouve aussi ces éléments dans les écrits sur l'AP (Gardner, 2002; Kelly \& Johnston, 2001). Ceci dit, chaque groupe focalisé présente des spécificités dans sa conception de l'AP.

Les intervenantes qui travaillent auprès de femmes. Ces intervenantes voient un lien étroit entre l'AP et la violence conjugale ou familiale. En cas de séparation des parents, l'AP serait souvent un prolongement d'une telle dynamique de violence:

Le terme [AP] lui-même, c'est une conséquence vraiment de la violence conjugale. [...] Dans presque tous les cas [d'AP], il y a eu de la violence physique grave sur la femme et il y a eu des actes physiques aussi sur l'enfant.

Dans cette conception, les mères pourraient utiliser de stratégies aliénantes pour protéger leurs enfants d'un homme qu'elles perçoivent comme dangereux, quitte à exagérer les faits dans certains cas:

Moi j'ai eu une femme dans les derniers mois. Elle, ce qu'elle a fait c'est qu'il y a un épisode de violence conjugale et elle a crié au loup dans le fond. Elle a dit toutes sortes de choses à la DPJ, aux policiers et il y a eu des conséquences à ça. Sauf qu'après, elle s'est rétractée et je pense qu'effectivement elle en a mis plus. [...] Sauf qu'elle s'est retrouvée piégée là-dedans elle aussi.

Quant aux pères, ils pourraient accuser leur ex-conjointe de faire de l'AP dans une tentative ultime de la contrôler ou de la punir d'être partie:

Il y a une situation présentement que le conjoint veut plaider pour aliénation parentale pour avoir la garde de l'enfant parce qu'il n'a plus aucune prise.

Ils pourraient également utiliser eux-mêmes de stratégies aliénantes auprès de l'enfant pour dénigrer leur ex-conjointe:

[La mère] avait déjà fait une dépression, elle avait été internée pendant un certain temps. [...] Ta mère est folle, puis tu ne peux pas rester avec, parce qu'elle est folle.

Certains auteurs ont mis ce genre de situation en lumière (Dalton, 1999; Gagné et al, 2005; Jaffe \& Geffner, 1998; Sachs, 2000) et incitent les intervenants et intervenantes psychosociaux et légaux à évaluer soigneusement la présence préalable de violence conjugale ou familiale lors d'allégations d'AP ou de comportements parentaux aliénants.

Les intervenants et intervenantes qui travaillent auprès d'hommes. Ces intervenants et intervenantes mettent davantage l'accent sur la position précaire des pères dans les familles, notamment lorsque le couple est en conflit. Lors d'un tel conflit, les intervenants et intervenantes pensent que le père serait désavantagé face à la mère dans une société où sont véhiculés des «stéréotypes » voulant que la mère soit l'experte de l'enfant:

Culturellement les hommes ne se sentent pas compétents dans leur rôle de père. 


\section{L'ALIÉNATION PARENTALE}

Il y a beaucoup de gars qui sont convaincus justement de cette incompétence là, pis qui s'effacent. [...] Ils s'aliènent eux-mêmes, là. Ils acceptent le stéréotype, pis c'est encore très vrai même chez les jeunes pères.

Ce faible sentiment de compétence amènerait parfois le père à négliger son lien avec l'enfant en laissant plus de place à la relation mère-enfant, le rendant très vulnérable à l'aliénation. On sait que l'exercice de la paternité à la suite d'une séparation se bute à plusieurs obstacles d'ordre personnel, social et juridique (Quéniart \& Rousseau, 2004). Dans une perspective plus préventive, il existe tout un corpus de littérature qui souligne l'importance de promouvoir l'engagement paternel dès les premiers moments de la vie de l'enfant (Turcotte, Dubeau, Bolté, \& Paquette, 2001). Ces préoccupations rejoignent les propos des intervenants et intervenantes qui œuvrent auprès des pères dans la présente étude.

Les intervenantes qui travaillent auprès de familles en difficulté. Ces intervenantes mettent l'accent sur la dimension psychologiquement violente des conduites parentales aliénantes. Il est clair pour elles que si les deux parents sont en conflit sans impliquer l'enfant, on ne parle pas d'AP. Par contre, lorsque l'enfant est impliqué dans le conflit, les intervenantes trouvent très difficile de faire la différence entre la triangulation de l'enfant dans le conflit et l'AP proprement dite:

Encore là, ça ne veut pas dire qu'il y a aliénation parentale dans cette famille-là. Il y a conflit entre les deux parents. Il y a triangulation de l'enfant, mais est-ce qu'il y a aliénation parentale? C'est encore la définition des concepts qui n'est pas claire.

Selon certains auteurs, la «triangulation » de l'enfant renvoie au fait de subir des pressions des deux parents pour choisir son camp ou de jouer le rôle d'espion ou de messager entre les deux parents (Grych, Seid, \& Fincham, 1992). Quoi qu'il en soit, les intervenantes de ce groupe jugent que ce genre de comportement parental constitue une forme de violence psychologique envers l'enfant dont les manifestations sont, par exemple, de dénigrer injustement l'autre parent devant l'enfant ou d'obliger l'enfant à choisir l'un de ses parents:

Je trouve que de faire ça à un enfant, le fait de le trianguler, le fait de dénigrer son autre parent et de lui faire vivre ça, c'est ça de la violence psychologique.

Ces propos rejoignent l'opinion de Gagné et Drapeau (2005) qui suggèrent que plusieurs conduites parentales aliénantes s'apparentent à de la violence psychologique envers l'enfant.

Les intervenants et intervenantes en protection de la jeunesse. Ces intervenants et intervenantes pressentent qu'il y a un processus, des étapes qui mènent à l'AP. Ils constatent que les stratégies aliénantes et le dénigrement tendent à s'amplifier au sein de la famille, jusqu'à un point de non retour. Un intervenant mentionne qu'il serait important

qu'on reconnaisse qu'il y a des étapes dans un contexte d'aliénation. [...] Y a un processus qui, je pense, t'as pas le choix-là, tu sais, ça s'en va vers le irréversible.

L'AP débuterait par un conflit conjugal accompagné d'une volonté d'effacer l'autre parent, de le discréditer aux yeux de l'enfant. Dans ce contexte, l'enfant vivrait de l'ambivalence qui se traduirait par un conflit de loyauté. L'enfant qui résoudrait ce conflit en rejetant complètement l'un de ses parents serait, finalement, aliéné. Cette définition de l'enfant aliéné correspond assez bien à celle proposée 
par Kelly et Johnston (2001)—d'autant plus que, à l'instar de ces auteures, les intervenants et intervenantes de ce groupe considèrent que les conflits chroniques qui caractérisent la famille qui vit de l'AP pourraient s'étendre à la famille élargie. Concernant l'enfant, un intervenant indique que

rendu à la fin, [...] t'es d'un bord, t'existes, t'es avec nous autres; t'es de l'autre bord, t'existes plus.

[...] C'est deux clans ennemis, là.

L'enfant devrait alors choisir l'un ou l'autre «clan » familial. Il pourrait éventuellement changer de clan et rejeter le parent avec lequel il s'était aligné au départ. Selon certains auteurs, ce genre d'alliance ambivalente, temporaire et réversible pourrait caractériser les enfants plus jeunes (4 à 8 ans) (Johnston \& Roseby, 1997).

Justement, les intervenants et intervenantes communautaires «famille » et en protection de la jeunesse sont d'avis que la propension d'un enfant à être aliéné pourrait dépendre de son stade de développement. Ainsi, un enfant en particulier pourrait être aliéné plus fortement que ses frères et sœurs. À leur avis, les plus âgés seraient moins à risque de devenir aliénés en raison de leur sens critique et de leur capacité de jugement plus développés. Pourtant, dans une analyse développementale de l'AP, Johnston et Roseby (1997) soutiennent que c'est l'enfant de 9 à 12 ans qui serait le plus vulnérable à l'AP. Il manque de distance critique pour bien évaluer la situation lorsqu'il est confronté à deux perspectives très différentes. Il risque alors de vivre très fortement le conflit de loyauté, qu'il résout en adoptant une vision réductrice de la situation et en décidant qu'un des parents est bon et que l'autre est mauvais. Contrairement aux enfants plus jeunes, cette cohorte adopterait des alliances irréversibles et davantage définitives. Quoi qu'il en soit, des études empiriques seront nécessaires pour déterminer l'influence de l'âge de l'enfant sur le développement de l'AP.

En somme, la vision qu'ont les intervenants et intervenantes concernant le concept d'AP est semblable en plusieurs points à celle relevée dans les écrits. Chaque groupe attire l'attention sur un aspect spécifique à considérer, soit le rapport avec la violence familiale et conjugale, notamment la violence psychologique envers l'enfant; soit la question de l'engagement paternel et de la valorisation du rôle de père; soit l'idée d'un processus d'AP et de l'influence du stade de développement de l'enfant sur l'AP. Tous ces aspects ont été relevés dans la littérature et reflètent des préoccupations importantes que la recherche future devra contribuer à clarifier.

\section{Les enjeux soulevés par la reconnaissance de l'AP}

Les intervenants et intervenantes rencontrés sont, de façon générale, en faveur d'une reconnaissance sociale et professionnelle de l'AP. Un point mentionné dans tous les groupes focalisés est qu'une telle reconnaissance pourrait entraîner des recours légaux plus adaptés pour les familles en difficulté, ce qui permettrait aux intervenants et intervenantes de les aider adéquatement. Les intervenants et intervenantes de tous les groupes jugent difficile de protéger les enfants qui vivent de l'AP au Québec parce qu'au moment où les groupes focalisés ont eu lieu la Loi sur la protection de la jeunesse ne reconnaissait pas la maltraitance psychologique comme un motif de compromission du développement de l'enfant. Cependant, un intervenant précise que le projet de loi $\mathrm{n}^{\circ} 125$, entériné récemment par l'Assemblée nationale, vise à actualiser cette reconnaissance: 


\section{L'ALIÉNATION PARENTALE}

Ils [le gouvernement] veulent inclure une nouvelle clause qui dit que la violence psychologique maintenant va pouvoir être un motif pour retenir un signalement. J'ai bien l'impression que l'aliénation parentale, les conflits entre les parents ça va rentrer dedans².

Les intervenants et intervenantes de tous les groupes indiquent aussi que la reconnaissance de l'AP aiderait à identifier les situations où la mère utilise les fausses allégations d'abus sexuel dans le but d'éloigner le père de son enfant. Selon eux, un tel geste pourrait s'expliquer par le désir de la mère de protéger son enfant s'il est victime de violence physique ou psychologique de la part de son père, ou si elle croit qu'il en est victime:

Elle s'est dit si je l'accuse [le père] d'abus sexuel, il ne verra plus ses enfants. C'est comme si par ce seul moyen-là je vais les protéger.

Les intervenants et intervenantes indiquent qu'une autre motivation serait la volonté d'obtenir l'exclusivité de la garde de l'enfant:

Des fausses allégations sont portées très souvent aux pères. [...] Pis quand la mère veut exclure le père, ben, elle fait des fausses allégations.

Une telle généralisation s'avère toutefois injustifiée lorsque l'on tient compte des connaissances disponibles sur les fausses allégations d'abus sexuel déjà mentionnées. Il reste que les intervenants et intervenantes des divers groupes trouvent difficile de discerner les fausses allégations des vraies dans une situation familiale très conflictuelle.

Les intervenants et intervenantes qui œuvrent auprès d'hommes et en protection de la jeunesse indiquent que la reconnaissance de l'AP permettrait de faire de la prévention:

Quand ça va être bien campé, on va être capable de voir les étapes avant que ça arrive, [...] on va prévenir ça. Parce que quand c'est rendu de l'aliénation parentale, c'est rendu très difficile d'intervenir.

Il est intéressant de remarquer que les intervenants et intervenantes en protection de la jeunesse introduisent le concept de prévention alors qu'ils interviennent typiquement dans des contextes de crise où le problème est souvent bien installé chez les familles. Le fait de voir des cas extrêmes et de constater les défis presque insurmontables qu'ils posent à l'intervention semble sensibiliser les intervenants et intervenantes à la nécessité de la prévention.

Une réserve des intervenants et intervenantes travaillant auprès de femmes et d'hommes est que la reconnaissance de l'AP pourrait amener une appropriation du concept par un groupe d'intérêt:

Mais c'est un débat [l'AP] [...] où il y a des groupes qui s'affrontent avec des idéologies qui peuvent être très opposées. [...] Ça enlève la crédibilité du juste milieu. Pis des questions qu'on doit se poser.

Ainsi, les intervenants et intervenantes considèrent qu'un groupe d'intérêt féministe ou masculiniste pourrait utiliser le concept afin d'accuser le parent du sexe opposé d'être aliénant. Le débat pourrait alors s'éloigner des questions de fond telles que gérer et résoudre les conflits familiaux.

Il semble toutefois paradoxal que les groupes d'intervenants et intervenantes qui expriment cette réserve soient aussi ceux et celles qui tendent à attribuer un genre au parent aliénant, et donc à orienter le discours vers un tel débat. Il est possible que ces intervenants et intervenantes vivent eux-mêmes un conflit de loyauté lorsqu'il est question d'AP: d'une part, ils désirent continuer à jouer leur rôle de 
défense de droits auprès des femmes/mères ou des hommes/pères, mais d'autre part, ils reconnaissent que les enfants sont vulnérables et qu'il est important de minimiser les conflits qui les affectent. Cette position inconfortable explique sans doute pourquoi leur discours peut sembler contradictoire. Afin de diminuer les tensions sociales existantes autour de l'AP, il devient essentiel de développer un discours plus scientifique, basé sur des données empiriques.

\section{Préférences d'intervention et besoins des intervenants et intervenantes}

Plusieurs interventions sont utilisées par la majorité des intervenants et intervenantes des groupes focalisés en matière d'AP. Parmi celles-ci, il y a le debriefing avec un parent ou un enfant qui a pour but de lui permettre d'évacuer ses émotions:

Tu veux ventiler, ventile [...] lâche toi «lousse ». [...] Ça aide à retrouver l'équilibre. Une fois les idées, la pression descendue un peu, les idées sont un peu plus en place.

Une autre intervention consiste à recadrer les responsabilités des acteurs familiaux afin de réduire la culpabilité de l'enfant et à permettre au parent de prendre conscience des responsabilités qu'il a envers lui:

C'est que les enfants disent c'est à qui la responsabilité quand papa dit des gros mots. [...] De pouvoir remettre les responsabilités de chacun [...] ça déculpabilise beaucoup l'enfant.

Le partenariat est une autre modalité d'intervention valorisée par tous les groupes rencontrés. Il peut être établi avec différentes personnes dans l'entourage de l'enfant comme un membre de la famille, les écoles, les services de garde, ou encore avec des intervenants et intervenantes du CLSC, d'organismes communautaires, de la police ou du système judiciaire. Selon les participants et participantes, l'intersectorialité et l'interdisciplinarité permettraient de voir le problème plus globalement, de mieux l'analyser pour faire des interventions adéquates et de se soutenir mutuellement dans leurs interventions.

Tous les participants et participantes soulignent qu'ils ont besoin de connaissances théoriques en matière d'AP. Ils indiquent qu'un modèle théorique plus précis et moins controversé pourrait aider à comprendre les fondements et les étapes de l'AP:

Juste de comprendre des fois, les fondements et d'amener peut-être après ça dans nos interventions, amener la personne à comprendre le pourquoi peut-être qu'elle fait ça.

De telles informations pourraient aussi permettre aux intervenants et intervenantes de mieux comprendre la signification des termes utilisés en cas d'AP et ainsi éviter la confusion entre les différents concepts.

Les intervenants et intervenantes qui travaillent avec les femmes, les hommes et en protection de la jeunesse mentionnent aussi avoir besoin de formation pratique afin de mieux dépister une situation d'AP et d'intervenir:

Sur ce que c'est, en quoi ça consiste, comment tu interviens? Qu'est-ce que tu fais, qu'est-ce qui faut que tu vérifies, qu'est-ce que tu ne fais pas?

Les outils dont les intervenants et intervenantes auraient besoin semblent plus spécifiques aux particularités de la clientèle avec laquelle ils interviennent. Les intervenantes qui travaillent auprès de 


\section{L'ALIÉNATION PARENTALE}

femmes indiquent qu'une grille permettant l'évaluation du parent aliéné leur serait utile, car elles ont le sentiment que certaines mères de leur clientèle se retrouvent dans cette situation. Les intervenants et intervenantes qui travaillent auprès d'hommes soulignent le besoin d'offrir aux pères des ateliers de compétence parentale. Ces ateliers donneraient confiance au père et, en cas d'AP, lui permettraient de rester dans la relation avec son enfant plutôt que de s'effacer au profit du parent aliénant. Les intervenantes qui travaillent auprès des familles ont mentionné plusieurs outils dont elles auraient besoin pour intervenir avec chacun des acteurs familiaux. Concernant les enfants, elles indiquent que des outils spécifiques à l'AP tels que des livres d'histoire, des poupées et des vidéos éducatives seraient utiles pour intervenir. Pour ce qui est des parents, elles indiquent qu'il devrait y avoir une formation offerte lors d'une séparation. Suite à cette formation, les parents seraient mieux outillés pour se séparer sans envenimer les conflits. Une telle formation pourrait donc être un moyen de prévenir l'AP.

\section{SYNTHÈSE DES GRANDS CONSTATS}

Cette recherche qualitative débouche sur trois grands constats concernant l'AP:

1. Aucun groupe d'intervenants et intervenantes n'a remis en doute l'existence du phénomène. Tous semblent reconnaître que cette problématique peut se retrouver dans un contexte de séparation et de conflits intenses et jugent qu'il est important d'intervenir dans une situation d'AP.

2. Il y a de nombreuses convergences dans les discours des différents groupes rencontrés. L'écart le plus grand semble se situer entre le groupe «femmes » et le groupe « hommes », car leur analyse du phénomène d'AP est fondée sur des idéologies différentes, voire opposées, comme le mentionne un participant. Il reste quand même plusieurs zones d'accord, suggérant que les discours extrémistes ont été peu ou pas représentés à l'intérieur de cette étude. Une première convergence concerne le cœur d'une définition de l'AP: une problématique d'ordre systémique liée à un conflit de couple lors d'une séparation ou d'un divorce. Une autre convergence retrouvée dans les résultats est l'importance d'avoir des recours légaux adaptés aux situations d'AP. Une dernière convergence concerne le manque de connaissances et d'outils, ce qui rend difficile l'évaluation et l'intervention dans des cas d'AP. Ainsi, sur la base de ce qu'ils savent et pressentent déjà, les intervenantes et intervenants doivent être alimentés et soutenus dans leurs actions auprès des familles par des activités de formation et de transfert de connaissances. Il semble clair que le développement de la recherche et de l'intervention devront aller de pair dans ce domaine et auront avantage à s'alimenter l'un l'autre.

3. Chaque groupe d'intervenants et intervenantes contribue à la compréhension d'une facette importante du phénomène de l'AP. Ses liens avec la violence familiale et conjugale sont soulignés, l'importance de promouvoir le rôle et l'engagement paternel est affirmée et l'idée de l'existence d'un processus d'AP est mise de l'avant. Ces éléments tendent à être complémentaires plutôt que mutuellement exclusifs. Ils permettent de mettre en perspective la problématique d'AP et d'envisager différentes trajectoires d'apparition et d'évolution de cette problématique. Une facette de la problématique que les participants et participantes n'ont pas mentionnée a trait à d'éventuels problèmes de santé mentale des parents, déclenchés ou amplifiés suite à une séparation difficile. 
Certaines études ont suggéré des liens entre l'AP et certains traits ou troubles de personnalité chez le parent aliénant (Baker, 2006; Lampel, 1996; Siegel \& Langford, 1998). Bref, l'AP pourrait bien présenter des déterminants et des trajectoires de développement multiples. Des études seront nécessaires pour examiner les différents cas de figure.

\section{CONCLUSION}

Il y a plusieurs acteurs dans une famille. Mère, père et enfants de même que les nouveaux conjoints et conjointes et la parenté élargie sont susceptibles de jouer un rôle dans une dynamique d'AP (Gagné et al., 2005). Pour les intervenants et intervenantes ayant participé à la présente étude, le fait d'adopter l'angle de vue de l'un ou l'autre de ces acteurs et de se centrer sur ses besoins permet de développer des savoirs pratiques spécifiques, mais complémentaires. D’ailleurs, ces intervenants et intervenantes souhaitent travailler davantage en partenariat dans les cas d'AP. Il serait intéressant de mettre sur pied un projet-pilote pour intervenir à plusieurs dans les situations d'AP, en assignant à chacun des acteurs familiaux une intervenante ou un intervenant chargé de le représenter. Chaque intervenante ou intervenant serait sélectionné en fonction de son expertise spécifique auprès des femmes, des hommes et des jeunes. Ils auraient le devoir de se concerter pour développer une compréhension commune de la situation, sensible aux réalités de chaque protagoniste, puis de proposer une intervention intégrée respectant autant que possible les besoins et les intérêts de chacun. Un tel projet-pilote devrait être suivi et évalué afin de s'assurer de la pertinence et de l'efficacité d'une telle approche.

Les forces de cette étude sont inhérentes à la diversification de l'échantillon, à la rigueur de l'analyse des données qualitatives et à l'emploi de la méthode du groupe focalisé. En plus d'être une méthode éprouvée permettant d'amasser une grande quantité de données dans un court délai (Morgan, 1988), celle-ci offre l'opportunité aux intervenants participants et intervenantes participantes de faire connaissance et de créer des liens. Cette étude comporte aussi certaines limites. La méthode des groupes focalisés permet un contrôle moindre sur les données recueillies que des techniques plus dirigées (Morgan, 1988). Il est également possible que certains participants et participantes aient ressenti une gêne ou un inconfort à exprimer leur opinion devant les autres.

Les résultats de cette étude descriptive et exploratoire suggèrent qu'il y a un grand besoin de continuer les recherches sur l'AP afin de soutenir les milieux de pratique dans leurs interventions. Cette étude se veut un point de départ sur lequel s'appuyer pour effectuer d'autres recherches qui permettraient de délimiter les frontières qui séparent les concepts de conflit, de violence conjugale, de maltraitance et d'AP. Il serait aussi utile d'étudier des trajectoires d'AP, afin d'en situer les étapes et les déterminants. Enfin, il sera crucial de préciser les symptômes, les réactions et les comportements des protagonistes qui font que l'enfant devient aliéné, de même que l'impact de l'AP sur le bien-être, la santé et l'adaptation des jeunes qui en sont la cible.

\section{NOTES}

1. (a) Une campagne de dénigrement, (b) des rationalisations non fondées, frivoles, voire absurdes, (c) une absence d'ambivalence, (d) une affirmation par l'enfant que sa décision de rejeter l'autre parent est la 


\title{
L'ALIÉNATION PARENTALE
}

sienne et et qu'il n'y a aucune contribution ou influence de la part de son parent aliénant, (e) soutien mutuel et inconditionnel de l'enfant et son parent préféré dans le conflit parental, (f) l'absence de culpabilité de l'enfant concernant la cruauté et/ou l'exploitation faite envers le parent aliéné, (g) utilisation par l'enfant pour exprimer sa colère envers le parent rejeté des mots, expressions ou phrases qui sont le reflet des termes véhiculés par le parent dit «aliénant», (h) des manifestations d'animosité et de rejet de l'enfant pouvant s'étendre à la famille élargie et au réseau social du parent ciblé.

2. La loi révisée est entrée en vigueur le 9 juillet 2007 et rend explicitement possible les signalements d'enfants à la direction de la protection de la jeunesse pour le motif de «mauvais traitements psychologiques ».

\begin{abstract}
This qualitative study explores the point of view of psychosocial practitioners regarding parental alienation (PA), which pertains to situations where a child rejects one of his or her parents under the influence of the other parent. Participants were practitioners from community organizations and social service agencies, working with women, men, children, and families. Four 2-hour long semistructured focus groups were conducted. The findings indicate that all participants recognized the existence of the PA phenomenon. Moreover, there were more convergences that expected between the discourses of the various groups. Also, each group of practitioners contributed to the understanding of an important aspect of PA. These findings indicate the need for future research aimed specifically at developing better knowledge and understanding of PA, which in turn could make it possible to help affected families by developing responses that are better adapted to their needs.
\end{abstract}

\section{RÉFÉRENCES}

Baker, A J.L. (2006). Patterns of parental alienation syndrome: a qualitative study of adults who were alienated from a parent as a child. American Journal of Family Therapy, 34, 63-78.

Brown, T., \& Frederico, M. (2000). Revealing the existence of child abuse in the context of marital breakdown and custody disputes. Child Abuse \& Neglect, 24, 849-859.

Campbell, T.W. (2005). Why doesn't parental alienation occur more frequently? The significance of discrimination. American Journal of Family Therapy, 33, 365-377.

Clawar, S.S., \& Rivlin, B.V. (1991). Children held hostage: Dealing with programmed and brainwashed children. Chicago: American Bar Association Press.

Dalton, C. (1999). When paradigms collide: Protecting battered parents and their children in the family court system. Family and Conciliation Courts Review, 37(3), 273-296.

Drapeau, S., Gagné, M.-H., \& Hénault, R. (2004). Conflits conjugaux et séparation des parents. Dans M.-C. SaintJacques, D. Turcotte, S. Drapeau, \& R. Cloutier (dir.), Séparation, monoparentalité et recomposition familiale: bilan d' une réalité complexe et pistes d'actions (pp. 175-194). Québec, QC: Presses de l'Université Laval.

Dunne, J., \& Hedrick, M. (1994). The parental alienation syndrome: An analysis of sixteen selected cases. Journal of Divorce and Remarriage, 21, 21-38.

Ellis, E.M. (2005). Help for the alienated parent. American Journal of Family Therapy, 33, 415-426.

Emery, R.E. (1999). Marriage, divorce, and children's adjustment ( $2^{\mathrm{e}}$ éd.). Thousand Oaks, CA: Sage.

Faller, K.C. (1998). The parental alienation syndrome: What is it and what data support it? Child Maltreatment: Journal of the American Professional Society on the Abuse of Children, 3, 100-115.

Gagné, M.-H., \& Drapeau, S. (2005). L'aliénation parentale est-elle une forme de maltraitance psychologique? Dans M. Baurain (dir.), Divorce \& séparation: $n^{\circ}$ 3. L'aliénation parentale (pp. 29-42). Bruxelles, Belgique: Éditions Labor.

Gagné, M.-H., Drapeau, S., \& Hénault, R. (2005). L'aliénation parentale: un bilan des connaissances et des controverses. Psychologie canadienne, 46, 73-87.

Gagné, M.-H., Drapeau, S., Melançon, C., Saint-Jacques, M.-C., \& Lépine, R. (2007). Links between parental psychological violence, other family disturbances and children's adjustment. Family Process, 46, 523-542. 


\section{REVUE CANADIENNE DE SANTÉ MENTALE COMMUNAUTAIRE}

Gardner, R.A. (1992). The parental alienation syndrome: A guide for mental health and legal professionals. Cresskill NJ: Creative Therapeutics.

Gardner, R.A. (1998). Recommendations for dealing with parents who induce a parental alienation syndrome in their children. Journal of Divorce and Remarriage, 28, 1-23.

Gardner, R.A. (1999). Family therapy of the moderate type of parental alienation syndrome. American Journal of Family Therapy, 27, 195-212.

Gardner, R.A. (2002). Parental alienation syndrome vs. parental alienation: Which diagnosis should evaluators use in child-custody disputes? American Journal of Family Therapy, 30, 93-115.

Gardner, R.A. (2003). The judiciary's role in the etiology, symptom development, and treatment of the parental alienation syndrome (PAS). American Journal of Forensic Psychology, 21, 39-64.

Greenbaum, T.L. (1998). The handbook for focus group research (2 éd.). Thousand Oaks, CA: Sage.

Grych, J.H., Seid, M., \& Fincham, F.D. (1992). Assessing marital conflict from the child's perspective: The Children's Perception of Interparental Conflict Scale. Child Development, 63, 558-572.

Institut de la statistique du Québec. (2001). Portrait social du Québec: données et analyses. Récupéré en ligne le 13 juin 2006 de: http://www.stat.gouv.qc.ca/publications/conditions/pdf/port_soc2001-3.pdf

Institut de la statistique du Québec. (2004). Enquête québécoise sur le tabac, l'alcool, la drogue et le jeu chez les élèves du secondaire. Récupéré en ligne le 13 juin 2006 de: http://www.stat.gouv.qc.ca/publications/ sante/pdf2005/Raptabac2004c2.pdf

Jaffe, P.G., \& Geffner, R. (1998). Child custody disputes and domestic violence: Critical issues for mental health, social service, and legal professionals. Dans G.W. Holden, R. Geffner, \& E.N. Jouriles (dir.), Children exposed to marital violence: Theory, research and applied issues (pp. 371-408). Washington, DC: American Psychological Association.

Johnston, J.R. (1993). Children of divorce who refuse visitation. Dans C. Depner \& J.H. Bray (dir.), Nonresidential parenting: New vistas in family living (pp.109-135). Newbury Park, CA: Sage.

Johnston, J.R., \& Roseby, V. (1997). Parental alignments and alienation among children of high conflict divorce. Dans J.R. Johnston \& V. Roseby (dir.), In the name of the child: A developmental approach to understanding and helping children of conflicted and violent divorce (pp.193-218). New York: Free Press.

Johnston, J.R., Walters, M.G., \& Friedlander, S. (2001). Therapeutic work with alienated children and their families. Family Court Review, 39(3), 316-333.

Kelly, J.B., \& Johnston, J.R. (2001). The alienated child: A reformulation of parental alienation syndrome. Family Court Review, 39(3), 249-266.

Krueger, R.A., \& Casey, M.A. (2000). Focus groups: A practical guide for applied research. London: Sage.

Lampel, A.K. (1996). Children's alignment with parents in highly conflicted custody cases. Family and Conciliation Courts Review, 34, 229-239.

L’Écuyer, R. (1990). Méthodologie de l'analyse développementale de contenu: méthode GPS et concept de soi. Québec: Presses de 1'Université du Québec.

Merriam, S.B. (dir.). (2002). Qualitative research in practice: Examples for discussion and analysis. San Francisco: Jossey-Bass.

Morgan, D.L. (1988). Focus groups as qualitative research. Newbury Park, CA: Sage.

O'Leary, K.D., \& Moerk, K.C. (1999). Divorce, children and the courts: Evaluating the use of the Parental Alienation Syndrome in custody disputes. Expert Evidence, 7, 127-146.

Pires, A. (1997). Échantillonnage et recherche qualitative: essai théorique et méthodologique. Dans J. Poupart, J.-P. Deslauriers, L.-H. Groulx, A. Laperrière, R. Mayer, \& A. Pires, La recherche qualitative, les enjeux épistémologiques et méthodologiques (pp. 113-169). Boucherville, QC: Éditions Gaëtan Morin.

Quéniart, A., \& Rousseau, N. (2004). L'exercice de la paternité à la suite d'un divorce: un parcours semé d'obstacles. Dans M.-C. Saint-Jacques, D. Turcotte, S. Drapeau, \& R. Cloutier (dir.), Séparation, monoparentalité et recomposition familiale: bilan d'une réalité complexe et pistes d'actions (pp. 101125). Québec, QC: Presses de l'Université Laval.

Ritchie, J., \& Lewis, J. (2003). Qualitative research practice: A guide for social science students and researchers. London: Sage. 


\section{L'ALIÉNATION PARENTALE}

Sachs, N.P. (2000). Is there a tilt toward abusers in child custody decisions? Journal of Psychohistory, 28(2), 203-228.

Siegel, J.C., \& Langford, J.S. (1998). MMPI-2 validity scales and suspected parental alienation syndrome. American Journal of Forensic Psychology, 16(4), 5-14.

Simard, G. (1989). Animer, planifier et évaluer l'action: la méthode du «focus group ». Laval, QC: Mondia.

Stahl, P.M. (1999). Complex issues in child custody evaluations. Thousand Oaks, CA: Sage.

Sullivan, M.J., \& Kelly, J.B. (2001). Legal and psychological management of cases with an alienated child. Family Court Review, 39(3), 299-315.

Thoennes, N., \& Tjaden, P.G. (1990). The extent, nature, and validity of sexual abuse allegations in custody/ visitation disputes. Child Abuse \& Neglect, 14, 151-163.

Trocmé, N., \& Bala, N. (2005). False allegation of abuse and neglect when parents separate. Child Abuse \& Neglect, 29, 1333-1345.

Turcotte, G., Dubeau, D., Bolté, C., \& Paquette, D. (2001). Pourquoi certains pères sont-ils plus engagés que d'autres auprès de leurs enfants? une revue des déterminants de l'engagement paternel. Revue canadienne de psycho-éducation, 30, 65-91.

Van Gijseghem, H. (2005). L'aliénation parentale: points controversés. Dans M. Baurain (dir.), Divorce \& séparation: $n^{\circ}$ 3. L'aliénation parentale (pp. 13-27). Bruxelles, Belgique: Éditions Labor.

Vassiliou, D., \& Cartwright, G.F. (2001). The lost parents' perspective on parental alienation syndrome. American Journal of Family Therapy, 29(3), 181-191.

Vestal, A. (1999). Mediation and parental alienation syndrome: Considerations for an intervention model. Family and Conciliation Courts Review, 37(4), 487-503.

Witkin, B.R. (1984). Assessing needs in educational and social programs. San Francisco: Jossey-Bass. 